\title{
MODEL THINKING: AN APPROACH FOR COPING WITH AN INCREASINGLY COMPLEX WORLD
}

\author{
Saikou Y. Diallo \\ Virginia Modeling Analysis and Simulation Center \\ Old Dominion University \\ 1030 University Blvd. \\ Suffolk, VA, USA \\ sdiallo@odu.edu
}

\author{
Samarth Swarup \\ Biocomplexity Institute and Initiative \\ University of Virginia \\ 995 Research Park Blvd. \\ Charlottesville, VA, USA \\ swarup@virginia.edu
}

\begin{abstract}
We need to train the next generation of scientists, modelers and analysts to be technically competent and socially aware transdisciplinary collaborators. Model thinking and Modeling and Simulation can play an even more significant role in society if we expand our horizons beyond socio-technical problems and tackle broader challenges impacting the human condition. In this paper, we discuss transdisciplinary collaboration, inclusion and social awareness as three pillars that can form the basis for maintaining and increasing the relevance of our field in a world where technological change is outpacing our ability to manage and predict its impact on key aspects of life and society.
\end{abstract}

Keywords: transdisciplinary teams, inclusion, accessibility, fairness, values.

\section{INTRODUCTION}

In this paper, we propose a framework for training experts that are inherently transdisciplinary in their approach. We believe that such experts are needed to cope with the level of complexity and interconnectivity we are facing today. Furthermore, we believe that we are on the verge of a "crisis" with the combination of instability created by environmental challenges which lead to frequent and disruptive conflict and unmanaged and unregulated technological advances which accentuate existing socio-economic inequality. Real-world problems don't come with disciplinary labels. They require multiple perspectives. An example is disaster preparedness and response. When a disaster such as hurricane strikes, effective response requires science-based policy, technology that allows rapid mobilization of resources in the right place at the right time, and efficient organization and management of human resources. These require input from urban planning, climate science, computer science and engineering, sociology, economics, public health, and much more. To address these challenges we need to move beyond disciplinary boundaries and engage real-world problems at scale.

From a technological perspective, we need to design and build the kinds of tools that not just allow, but enhance this kind of collaboration (Barrett et al., 2011; Barrett et al., 2015). The process of design, implementation, and use also needs to involve all stakeholders, with a clear understanding of values (e.g., Borning et al.,(2004), Björgvinsson et al., 2010, Briot et al., (2007)). This is a view and methodology that has emerged over the last two decades and continues to develop through a rich discourse in the literature. 
However, there has been relatively little written about how to train the "makers" in this challenging process. That is the focus of this paper.

We adopt the term model thinking to describe our perspective. The goal is not to make modelers and analysts experts in every domain. Rather, the model thinking approach is to train them to do their work in a manner that gives primacy to transdisciplinary collaboration, inclusion, and social awareness. At the same time, we must create the ecology that enables the model thinking approach to succeed. We need to build a framework that supports model thinking practitioners by hiring, empowering, and promoting them.

Current training at universities and schools is centered around producing graduates who can stand on the sacred grounds of the discipline from which they receive training. Engineers are expected to acquire competency in the sub-area of engineering in which they are being trained (Electrical, Computer, Mechanical, etc.) and spend a career solving problems that belong to that area. Social scientists and humanists follow the same pattern and are considered successful when they master the canons of their discipline and contribute to it. Eventually, those who excel in their respective disciplines become experts and some of those experts act as gatekeepers and enforcers of the norms that guide future candidates. Experts can promote lines of thinking that they believe are most likely to create useful knowledge by acting as reviewers, serving on panels and teaching. The current system encourages and rewards each specialist and experts to stay close to the canons and focus on mastering a topic. While positive and beneficial, this approach can push experts to 1) apply disciplinary assumptions to reduce complex situations to problems that fit their discipline, 2) define the wrong problem and propose plausible solutions which might create unnecessary chases down rabbit holes and 3) fail to recognize the existence of a problem because it does not fit the canons of one's discipline. The first two points can be arguably addressed by better training but the third one is most closely connected to the expert's willingness to speak to an issue that they believe to be outside of their domain. This issue is commonly observed when engineers fail to engage with social issues and social scientists believe that they have little to offer in creating engineering solutions. While not explicitly discouraging radical transdisciplinary collaboration, the current approach to training prevents the types of collaborations needed to tackle the global challenges we are now facing. Issues such as migration, global warming, child sex trafficking transcend national boundaries, expertise and require an "all hands" approach that is difficult to achieve with our current approach.

The framework we propose is based on a few key observations:

- We operate on a spectrum where neurodiversity is the norm: People are different and unique, and they live in different and unique environments. Their perceptions of problems and solutions are shaped by their physical and neurocognitive aptitudes. Consequently, we must tackle problems with a diverse set of expertise that is reflective of the diversity of those who will be impacted by the solutions. Experts should operate and train to support and enable everyone to access and use solutions at every level.

- We must strive for universal design-solutions and tools that are equitable, flexible, simple, errortolerant and accessible with low physical effort. While this is arguably prohibitive in cost and time, experts should have to make the case for excluding a group rather that making the case for including one. The distinction between inclusive design and universal design is an important discussion but at a minimum, expert must be trained to create an impact assessment for what the problem that they are solving.

- Collaborative engagement is necessary to understand the needs of the communities that we aim to serve. Solutions designers are often keenly aware of the limitations of tools and resources and therefore tend to over-constrain problem specifications to the ones they believe they can solve. This might not or might not be most pressing problem for the community at that moment. The result is 
a scientifically interesting solution with little practical grounding. They are a plethora of solutions in modeling and simulation that are ignored by the community they purport to serve, This is particularly true in the policy domain because of the disconnect between the specialized technical language of simulation solutions and the need for highly contextualized solution options for policy makers.

- Solutions must be open and accessible not only to the specialized professional but to the interested general public. It is difficult to create trust without understanding. Researchers must open laboratories to educate the public on tools, methodology and processes. We must strive to integrate the general public, students at every level and industry members into daily operations. Technology, infrastructure and resources such as virtual reality, augmented reality, internet of things must be available to people across all communities regardless of training, age or physical restrictions

- It is important to communicate to future researchers the need and value of research that is impactful and responsible. The idea is to foster concerned researchers who use their gifts and training not only to advance science but primarily to improve the human condition. This requires a shift in focus from "research what is of interest to you" to "research what is beneficial to those around you". This requires us to revisit how we evaluate, hire and promote by allocating a higher value to community involvement. Rather than opposing evaluation models, it would be preferable to have multiple models that researchers can choose from at key moments in their career. For instance, an academiccentric model where the researcher advances fundamental knowledge can coexist with a community-centric model where the researcher is focused on solving the pressing needs of the community. This is somewhat reflected in the tenured versus research tracks but in practice the distinction in terms of evaluation and promotion is only evident to those directly involved. The community is not fully served by either in the current design. Students have very little opportunity to work across disciplines and often inherit the disdain or appreciation for other types of knowledge from the culture of their alma mater. Once set in a career, with the incentives heavily weighted towards disciplinary excellence it is unreasonable to expect a transdisciplinary approach to solving complex problems. It is therefore critical, to seek out diverse worldviews and conduct research that seeks out all disciplines and values expertise regardless of its origin. This can only be by design and must be incorporated in budgets and proposals with a fair distribution of resources across participants. Once awarded, projects constitute an opportunity to create transdisciplinary teams of students where they can learn to appreciate the practical value of the arts, humanities, social sciences and other disciplines that are typically under-represented in engineering projects.

The balance of the paper is organized as follows. In the next section we discuss the need for model thinkers across all disciplines to collaborate to solve complex problems at the global level. We then present a framework for developing, hiring and fostering model thinkers followed by a discussion on the special importance of social awareness and inclusion in section 3 . We conclude with a roadmap to implement the proposed framework in section 4.

\section{MODEL THINKERS AND SOCIETAL PROBLEMS}

Societal problems are too big and complex to be solved by individuals. Toy models of real-world problems rarely have the right kind of complexity to produce meaningful insights. We need to exploit the increasing amounts of data that are now available in multiple domains to construct detailed high-fidelity models that allow us to address problems at scale. Team science is needed for this kind of approach. Working effectively in teams is a skill that needs to be inculcated in academic research from early stages. Exposing undergraduates to cutting-edge research projects being done by large teams is essential in this process. 


\section{Diallo and Swarup}

How do we systematically train and foster model thinkers from high school through the workforce? We propose a framework for research institutes to hire, empower and promote (HEP) model thinkers. The HEP framework is an iterative knowledge generation process that seeks to 1) train future model thinkers by engaging them early on and incorporating them into the daily life of the center, 2) constantly seek out and respond to community needs by being aware and attempting to solve problems that are relevant to them, and 3) promote and empower the talented workforce by fostering a supportive, transdisciplinary ecosystem with the requisite variety or talent and project portfolio.

Figure 1 shows a process view of the HEP. We identify seven processes and six stakeholder groups. This framework provides a structured conception of the processes in a research organization that can support model thinking and the training of next generation modelers and analysts. It is important to point out that this framework is embedded in a larger context of interaction with students (Process 6) and the community (Process 5). This is to emphasize the role of social awareness, values, and inclusion in this framework.

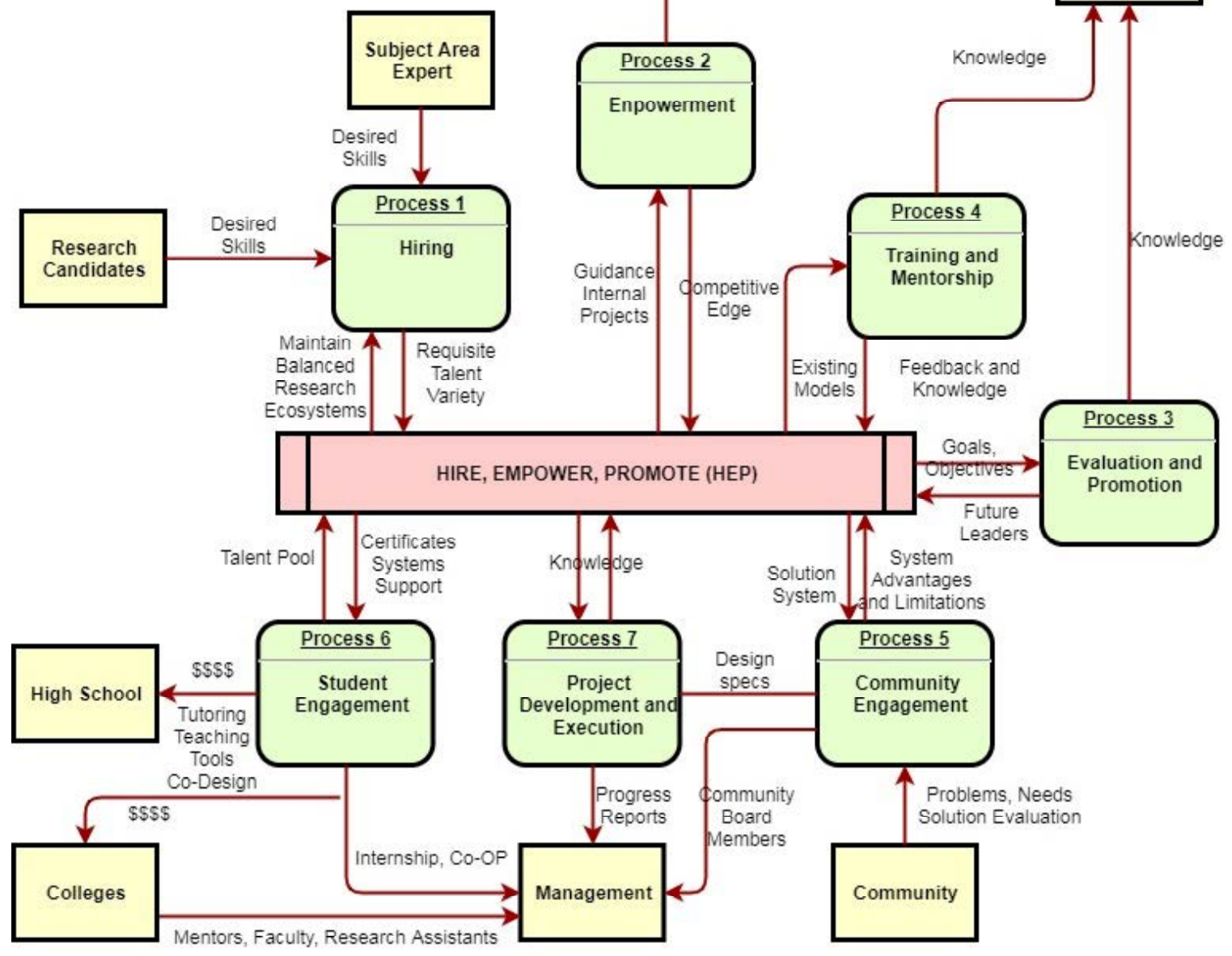

Figure 1: Hire Empower Promote (HEP) Process.

Figure 1 shows a process view of the HEP. The HEP framework identifies six stakeholder groups and seven processes that must interact at least once in an instantiation of the process. Community engagement is a 
critical component of this process. Community refers to organized groups or individuals who might be impacted by a solution developed by the center. The HEP recommends the creation of a diverse community board to help it identify problems, concerns and needs. The community engagement process (Process 5) seeks to systematically interact with its board but also stay aware of problems and issues at the regional, national and international level. The result of the engagement process is a documented set of issues that the center aims to explore with its partners through the project development and execution process (Process 7).

The project development and execution process identify requirements, needs and target stakeholders. It must incorporate a plan to engage students in high school as well as colleges. Management ensures that the project is well-balanced in its design and expertise pool. Each project is an opportunity for future model thinkers to interact together and with mentors from multiple backgrounds. IT is also an opportunity to acquire talent and expertise in areas that are lacking. That expertise is identified early in during the student engagement process (Process 6). While the tendency is to reward the "best student" or the most "skilled student", it is critical to value collaboration and effective team building over technical skill. It is also essential to seek out students from backgrounds outside the traditional pool. Students on the hearing, vision or autism spectrum force an adaptation in the work environment that forces others to challenge preconceived notions. Those students also represent worldviews that are not usually considered in the project development and engagement process. As a result, we expect a culture of fair and inclusive solution design to become second nature to the organization.

The hiring process (Process 1) must therefore balance the needs for talent and expertise with the current project pool and the community needs. Rather than hire around projects, HEP promotes hiring around balance, collaboration and team. In HEP, hiring means 1) bringing people into the organization on a fulltime basis, 2) bringing individual experts when needed and 3) engaging a community of vendors to fill highly specialized needs. The vendor community and individual experts make up the subject area expert pool that the organization relies on to execute projects or seek funding to address the needs of the community.

Empowerment (Process 2), training and mentorship (Process 4) and evaluation and promotion (Process 3) constitute the social tissue that enables the organization to effectively function to respond to the needs of its community. They aim to keep all stakeholder groups engaged and the collaborating. Each process requires different strategies vary from stakeholder to stakeholder. From the outset of this paper, we insisted on individual differences and thinking in spectrums. As a result, each research center must adapt its processes to their ecosystem and create a range of models for these processes. Anecdotally, research centers are very aware of the need to regularly update these processes to reflect the changing landscape of talent, projects and funders. The goal of these processes to create a pipeline of researchers who are trained and mentored to respond to the needs of the world around them and are motivated by mentors and colleagues who set the example. In the next section, we illustrate how the HEP framework can be used to address societal problems through simulation and analytics

\section{SOCIAL AWARENESS, VALUES, AND INCLUSION}

Being a model thinker also means understanding the role of the models and tools we build in society, including their use and potential misuse. Social simulations have a special role in the broader ecosystem consisting of information and communication technologies, their designers, their users, and their indirect stakeholders. While Big Data tools have entered the mainstream and have begun to be widely used by individual users (e.g., GPS-based navigation with live traffic conditions), large-scale simulations and related tools are still in the domain of academic and (to some extent) governmental or institutional use. This is because simulations are often used for informing policy decisions (Barrett et al., 2011), thereby impacting large populations and infrastructures, with or without their awareness. This puts an ethical and moral burden 
on the makers and users of these tools. As students learn to be experts in modeling and simulation, we must also prepare them for a future where simulation and modeling become mainstream and are used by a much broader class of users.

Some of the broader issues simulation modelers must consider in the process of creating adequate (Swarup, 2019) systems are:

- Who chooses the question?

- Who builds the system?

- Who uses it?

- Who is impacted by it?

The question which the system is created to answer is in some sense the most important element of the specification and design of the solution. The way that a question is framed can set up expectations about admissible solutions. For example, the questions "how do we reduce illegal immigration?" and "how do we strengthen border security?" are ostensibly about the same problem, but the way they are stated already implies two different spaces of answers with different (though overlapping) underlying value assumptions.

In real-world applications, the people who choose the question are generally not the people who design and build the computational system to answer it. This raises the possibility of value conflicts between the two groups and, unless we have an explicit process for identifying values, these conflicts can remain hidden or unknown. The resulting system or tool would then have a subtle mismatch with the specifications. This is not to say that the client is always right when it comes to values. The process of identifying values and making them explicit may also help the people choosing the question to refine or reframe it. In academic/research applications, it is more often the case that the people who choose the question are the same as the people building the system. Here, too, it is important go through a process of value identification in order to achieve an explicit consensus around the goals and methods for solution design and implementation.

Frameworks have emerged to address these challenges, such as value-sensitive design (e.g., Borning et al., 2004), participatory design (e.g., Björgvinsson et al., 2010), and participatory simulation (e.g., Briot et al., 2007). All these frameworks recognize the importance of including all stakeholders in the design and use of simulation tools. They differ in the process by which stakeholders are involved, and the stage of the development process in which they are involved.

Value-sensitive design (VSD) was developed in the context of the UrbanSim simulation of land use (Waddell et al., 2003). VSD follows a tripartite methodology, involving conceptual, empirical, and technical investigations, where the goal is to systematically identify the stakeholders, elicit values from them, and determine how these can be designed into the system. For example, in the case of UrbanSim, they were able to identify and interact with several important stakeholders, including elected officials, staff at government agencies, businesses in the region, workers, neighborhood groups, and more. Through this process, they identified several values such as environmental sustainability, neighborhood walkability, affordable housing, minimal governmental intervention, etc. They also committed to three moral values: fairness, accountability, and democracy (Borning et al., 2004).

In order to make the UrbanSim tool usable and useful, they developed a series of indicators that provided feedback to the stakeholders about how their choices would affect they things they value (Davis 2008). When used in this manner, therefore, UrbanSim becomes a part of the cognitive loop of users, providing them with a new affordance: allowing them to understand the long-term consequences of their choices in terms of their values. By its nature, this is an ongoing social cognitive process, where the tool acts as infrastructure facilitating interaction (Barrett et al., 2015). 
This perspective also broadly matches the idea of participatory design. In one interpretation, Björgvinsson et al. (2010) discuss how participatory design can be viewed as a combination of "Things and infrastructuring". To them, infrastructure is not a platform on which other applications run. Rather, it is "an ongoing alignment of contexts," a concept earlier explicated by Star and Ruhleder (1996), who discuss infrastructure in relational terms. Similarly, things, in the sense of Björgvinsson et al. (2010) are "innovation milieus," relational systems of which computational tools are a part. This primacy of process is central to the relational perspective. They realize this perspective through Living Labs, which are spaces organized around the principles of involving stakeholders in the design process and doing experimentation in realworld settings (Almirall \& Wareham, 2011).In addition to involving stakeholders in the design process, they can also be involved directly in the simulation itself, through a process of participatory simulation (Briot et al., 2007). This approach combines agent-based simulations with role-playing games. Multiple participants interact in a structured game-like environment, which is also responsible for computing the consequences of their actions through a simulation. This approach has been applied to situations involving complex decision-making and multiple active stakeholders, such as management of protected areas (Briot et al., 2007), and Chesapeake Bay restoration (Learmonth et al., 2011).

The common theme that emerges from all these approaches is the importance of involving people in the development of models and tools. A model is not just the representation in the computer, it is an extended thing encompassing computational tools, their makers, and their users (Barrett et al., 2015). In order to train "model thinkers," we must create a curriculum that recognizes this extended relational perspective and creates opportunities for students to participate in large-scale research and design projects with community involvement.

There are also several open questions and challenges in this field. One such challenge is that values themselves evolve over time. How do we recognize and integrate such emergent values (Le Dantec et al., 2009), and what do we do if some stakeholders are deceptive or malicious? Similarly, how do we verify that a given complex model or tool actually (correctly) instantiates the values claimed by the designers? If every stakeholder is a decision-maker, how do we ensure that the resulting decisions are cooperative and (pareto-)optimal? Ultimately the solutions are not technological alone, but are, like the processes themselves, relational and ongoing.

\section{CONCLUSIONS}

We believe that some fundamental changes are needed in the processes by which we train modelers, analysts, and makers in general. The old paradigm of focusing on excellence in one domain needs to give way to a new paradigm which emphasizes transdisciplinarity, inclusion, and social awareness, an approach we call model thinking.

This is a fundamental change because it requires not just a new philosophy of the role of modelers and analysts in society, but also requires creating a new ecology that hires, empowers, and promotes people who are willing to dedicate significant effort to engaging with the community and working beyond their disciplinary boundaries in a value-focused way.

As we gather ever more data about individuals and communities, and as we create ever more sophisticated methods for building models and analyzing data, we must not lose sight of the fact that we also need to train the makers and users of these tools to work toward social and public good. Technology alone cannot solve complex social problems, unless it is coupled with social systems in the right way. It will be an ongoing complex process, and we believe model thinking is the guiding principle to steer us through it. 


\section{ACKNOWLEDGMENTS}

This work was partially supported by DTRA Grant HDTRA1-17-F-0118 and NASA grant $80 \mathrm{NSSC} 18 \mathrm{~K} 1594$. Any opinions, findings, and conclusions or recommendations expressed in this material are those of the authors and do not necessarily reflect the views, official policies or endorsements, either expressed or implied, of NASA, DTRA, or the U.S. Government.

\section{REFERENCES}

Almirall, E. \& Wareham, J. 2011. Living Labs: Arbiters of Mid- and Ground-level Innovation. Technology Analysis \& Strategic Management 23(1), 87-102.

Barrett, C. L., Eubank, S. G., Marathe, A., Marathe, M. V., Swarup, S. 2011. Information Integration to Support Model-based Policy Informatics. The Innovation Journal 16(1), Article 2.

Barrett, C. L., Eubank, S. G., Marathe, A., Marathe, M. V., Swarup, S. 2015. Synthetic Information Environments for Policy Informatics: A Distributed Cognition Perspective. In, Johnston, E. (Ed.), Governance in the Information Era: Theory and Practice of Policy Informatics, Routledge, 267-284.

Björgvinsson, E., Ehn, P., Hillgren, P.-A. 2010. Participatory Design and "Democratizing Innovation." Proceedings of the Participatory Design Conference.

Borning, A., Friedman, B., Kahn Jr., P. H. 2004. Designing for Human Values in an Urban Simulation System: Value Sensitive Design and Participatory Design. Proceedings of the Eighth Biennial Participatory Design Conference.

Briot, J.-P., Guyot, P., Irving, M. 2007. Participatory Simulation for Collective Management of Protected Areas for Biodiversity Conservation and Social Inclusion. AIS-CMS 7, 183-188.

Carnell, R. 2018. 1hs: Latin Hypercube Samples.

Chang, W. \& Ribeiro, B. B. 2018. shinydashboard: Create Dashboards with 'Shiny'.

Chang, W., Cheng, J., Allaire, J., Xie, Y. \& McPherson, J. 2018. Shiny: Web Application Framework for R.

Chen, T., He, T., Benesty, M., Khotilovich, V., Tang, Y., Cho, H., Chen, K., Mitchell, R., Cano, I., Zhou, T., Li, M., Xie, J., Lin, M., Geng, Y. \& Li, Y. 2018. Xgboost: Extreme Gradient Boosting.

Davis, J. 2008. Engaging and Informing Citizens with Household Indicators. Proceedings of the 41st Hawaii International Conference on System Sciences.

Gilbert, Nigel, Ahrweiler, Petra, Barbrook-Johnson, Pete, Narasimhan, Kavin Preethi and Wilkinson, Helen (2018) 'Computational Modelling of Public Policy: Reflections on Practice' Journal of Artificial Societies and Social Simulation 21 (1) 14. DOI: 10.18564/jasss.3669

Jager, W. \& Edmonds, B. (2015) Policy Making and Modelling in a Complex world. In Janssen, M., Wimmer, M. and Deljoo, A. (eds.) Policy Practice and Digital Science. Springer, pp. 57-74. DOI:10.1007/978-3-319-12784-2_4

Le Dantec, C. A., Poole, E. S., \& Wyche, S. P. 2009. Values as Lived Experience: Evolving Value-Sensitive Design in Support of Value Discovery. Proceedings of the CHI Conference.

Learmonth, G., Smith, D. E., Sherman, W. H., White, M. A. \& Plank, J. 2011. A Practical approach to the complex problem of environmental sustainability: The UVA Bay Game. The Innovation Journal: The Public Sector Innovation Journal 16 (1).

Lumley, T. 2017. Leaps: Regression Subset Selection. 


\section{Diallo and Swarup}

Millington, J. D. A., O'Sullivan, D. \& Perry, G. L. W. 2012. Model histories: Narrative explanation in generative simulation modelling. Geoforum; Themed issue: Spatialities of Ageing, 43, 1025-1034. doi: https://doi.org/10.1016/j.geoforum.2012.06.017.

Perry, G. L. W. \& O'Sullivan, D. 2018. Identifying Narrative Descriptions in Agent-Based Models Representing Past Human-Environment Interactions. Journal of Archaeological Method and Theory, 25, 795-817. doi: 10.1007/s10816-017-9355-x.

Prowse, T. A. A., Bradshaw, C. J. A., Delean, S., Cassey, P., Lacy, R. C., Wells, K., Aiello-Lammens, M., Akçakaya, H. R. \& Brook, B. W. 2016. An efficient protocol for the global sensitivity analysis of stochastic ecological models. Ecosphere, 7, e01238. doi: 10.1002/ecs2.1238.

Star, S. L., Ruhleder, K. 1996. Steps Toward an Ecology of Infrastructure: Design and Access for Large Information Spaces. Information Systems Research 7(1), 111-134.

Stein, M. 1987. Large Sample Properties of Simulations Using Latin Hypercube Sampling. Technometrics, 29, 143-151. doi: 10.1080/00401706.1987.10488205.

Swarup, S. 2019. Adequacy: What Makes a Simulation Good Enough? Proceedings of the Spring Simulation Conference (SpringSim).

Swarup, S., Marathe, A., Marathe, M. V., Barrett, C. L. 2019. Simulation Analytics for Social and Behavioral Modeling. In, Davis, P. K.; O'Mahony, A.; Pfautz J. (Eds.), Social-Behavioral Modeling for Complex Systems, pp. 617--632, Wiley.

Team-R-Core. 2015. R: A Language and Environment for Statistical Computing. Vienna, Austria: R Foundation for Statistical Computing.

Waddell, P., Borning, A., Noth, M., Freier, N., Becke, M. \& Ulfarsson, G. 2003. Microsimulation of Urban Development and Location Choices: Design and Implementation of UrbanSim. Networks and Spatial Economics 3, 43-67.

\section{AUTHOR BIOGRAPHIES}

SAIKOU Y. DIALLO is a Research Associate Professor at the Virginia Modeling, Analysis, and Simulation Center (VMASC) where he serves as the head of the Simulated Empathy lab. He is also an adjunct Professor of Modeling, Simulation, and Visualization Engineering at Old Dominion University (ODU). He received his $\mathrm{MS}$ and $\mathrm{PhD}$ in Modeling and Simulation from ODU. His email address is sdiallo@odu.edu and his web page is http://www.odu.edu/vmasc/research/simulated-empathy.html.

SAMARTH SWARUP is a Research Associate Professor at the Biocomplexity Institute and Initiative at the University of Virginia. He received his $\mathrm{PhD}$ in Computer Science from the University of Illinois at Urbana-Champaign. His email address is swarup@virginia.edu and his web page is 